\title{
Black Pain Matters: Prioritizing Antiracism and Equity in the Opioid Epidemic
}

\author{
Utibe R Essien, MD, MPH ${ }^{1,2 \star}$, Ayomipo Ifidon, BS', Kimberly L Sue, MD, PhD ${ }^{3}$
}

${ }^{1}$ Department of Medicine, University of Pittsburgh School of Medicine, Pittsburgh, Pennsylvania; ${ }^{2}$ Center for Health Equity Research and Promotion, VA Pittsburgh Healthcare System, Pittsburgh, Pennsylvania; ${ }^{3}$ Department of Internal Medicine, Yale School of Medicine, New Haven, Connecticut.

n 2016, a study was published that continues to shock observers today. ${ }^{1}$ Examining 200 medical trainees, researchers reported that an alarming percentage of these individuals held false beliefs about Black bodies, including 22\% believing that nerve endings in Black persons are less sensitive than nerve endings in White persons and 63\% believing that Black skin is thicker than White skin. Furthermore, the study found that those who held these false beliefs about biological differences between Black and White individuals were also less likely to recommend pain treatment to Black patients in a follow-up case vignette. Two years later, in an evaluation of racial differences in opioid prescribing in the United States published in Epidemiology, one of the authors suggested, "It's an extremely rare case where racial biases actually protected the population [Black individuals] being discriminated against."

These studies provide the background for the analysis by Rambachan et $\mathrm{al}^{3}$ published in this issue of the Journal of Hospital Medicine. The authors examined a diverse cohort of more than 10,000 patients hospitalized on a general medicine service at an academic medical center in San Francisco from 2012 to 2018. Black patients were significantly less likely to receive an opioid prescription at discharge, and when they did, were discharged on opioids for fewer days than White patients. No other racial group experienced such a disparity, with Asian patients more likely to receive opioids at discharge. Whereas these findings align with myriad studies demonstrating racial disparities in opioid prescribing, ${ }^{4}$ the authors focus on patients admitted to a general medicine service, where most hospitalized patients receive medical care daily.

The authors concede that determining the etiology of these disparities was beyond the scope of their study, yet this is the exact question we must answer today. Why should the color of a patient's skin continue to determine the type, and duration, of care they receive, especially when treating pain? The authors hypothesize that individual factors such as provider bias and systemic factors, including limited guidelines on pain management, may drive the observed racial inequities. This progression from individual- and institutional- to communityand policy-level determinants offers a useful framework for understanding the drivers of disparities in opioid prescribing. It also provides an agenda for future research that can guide us

*Corresponding Author: Utibe R Essien, MD, MPH; Email: uessien@pitt.edu.

Received: July 21, 2021; Revised: September 13, 2021;

Accepted: September 13, 2021

(C) 2021 Society of Hospital Medicine DOI 10.12788/jhm.3703 from simply detecting disparities to understanding and eliminating them. Furthermore, it is important to examine care team provider characteristics, including race/ethnicity, years in practice, education level (eg, resident vs attending), ${ }^{5}$ experience with implicit bias training, and differential referral to specialists, such as pain, palliative care, and addiction providers. Factors associated with the facility where a patient is hospitalized also warrant further exploration, including the diversity of medical and nonmedical staff as well as patients. ${ }^{6}$ Examining these factors will allow us to move closer toward implementing effective interventions that eliminate disparities in pain treatment.

The authors begin to provide us with possible levers to pull to address the inequities in opioid prescribing. They suggest provider-level bias training, improved institutional tracking of disparities, and policy-level solutions to address the persistent dearth of diversity in the healthcare workforce. While these broad solutions may address health disparities across the medical field, targeted solutions are needed to directly address inequities in pain treatment. First, we must explore the reasons for disparities in the prevalence, presentation, and management of pain in Black populations. These reasons may include occupational exposures or injuries, psychological stress (often associated with racism), and a disproportionate presence of chronic medical comorbidities. Second, health systems can implement a standardized system for opioid prescribing, supported by pharmacy expertise and considering clinical diagnoses, to reduce subjectivity associated with determining the appropriateness of an opioid prescription. Third, health systems must improve access to addiction, harm reduction, and pain specialty services to effectively manage comorbid conditions in at-risk patients. ${ }^{7}$ Furthermore, we must look beyond traditional measures of healthcare access, such as insurance coverage, to address social determinants of health, such as distance to pharmacy, housing security, employment status, and experience with the criminal justice system, which may influence a patient's receipt of a prescription. Finally, as a society, we must prioritize early training of healthcare providers, long before the undergraduate and graduate medical education level, to practice medicine without stigmatizing biases and stereotypes related to drug use in communities of color. ${ }^{8}$

The pattern of racial and ethnic disparities in healthcare has been documented for decades, with an ever-increasing depth of the different ways in which minoritized patients are undertreated. Despite this breadth of research, our understanding of the etiology of these inequities and development and implementation of interventions to reduce them remain limited. Rambachan et $\mathrm{al}^{3}$ do a commendable job highlighting further 
racial disparities in opioid prescribing in hospitalized patients and provide another opportunity to answer the important questions plaguing health care today: Why do these disparities exist and what can be done to address them? The urgency we take towards answering these questions will confirm our commitment to achieving antiracism in medicine and prioritizing health equity. Black lives are depending on it.

Disclosures: The authors reported no conflicts of interest.

\section{References}

1. Hoffman KM, Trawalter S, Axt JR, Oliver MN. Racial bias in pain assessment and treatment recommendations, and false beliefs about biological differences between blacks and whites. Proc Natl Acad Sci U S A. 2016;113(16):4296-4301. https://doi.org/10.1073/pnas.1516047113

2. Alexander MJ, Kiang MV, Barbieri M. Trends in Black and White opioid mor- tality in the United States, 1979-2015. Epidemiology. 2018;29(5):707-715. https://doi.org/10.1097/EDE.0000000000000858

3. Rambachan A, Fang MA, Prasad P, Iverson N. Racial and ethnic disparities in discharge opioid prescribing from a hospital medicine service. J Hosp Med. 2021;16(10):589-595. https://doi.org/10.12788/jhm.3667

4. Essien UR, Sileanu FE, Zhao X, et al. Racial/ethnic differences in the medical treatment of opioid use disorders within the VA healthcare system following non-fatal opioid overdose. J Gen Intern Med. 2020;35(5):1537-1544. https:// doi.org/10.1007/s11606-020-05645-0

5. Essien UR, He W, Ray A, et al. Disparities in quality of primary care by resident and staff physicians: is there a conflict between training and equity? J Gen Intern Med. 2019;34(7):1184-1191. https://doi.org/10.1007/s11606-019-04960-5

6. Hollingsworth JM, Yu X, Yan PL, et al. Provider care team segregation and operative mortality following coronary artery bypass grafting. Circ Cardiovasc Qual Outcomes. 2021;14(5):e007778. https://doi.org/10.1161/CIRCOUTCOMES. 120.007778

7. Sue KL, Fiellin DA. Bringing harm reduction into health policy - combating the overdose crisis. N Engl J Med. 2021;384(19):1781-1783. https://doi. org/10.1056/NEJMp2103274

8. James K, Jordan A. The opioid crisis in Black communities. J Law Med Ethics. 2018:46(2):404-421. https://doi.org/10.1038/jes.2015.55

\section{Journal of Hospital Medicine}

\section{journalofhospitalmedicine.com}

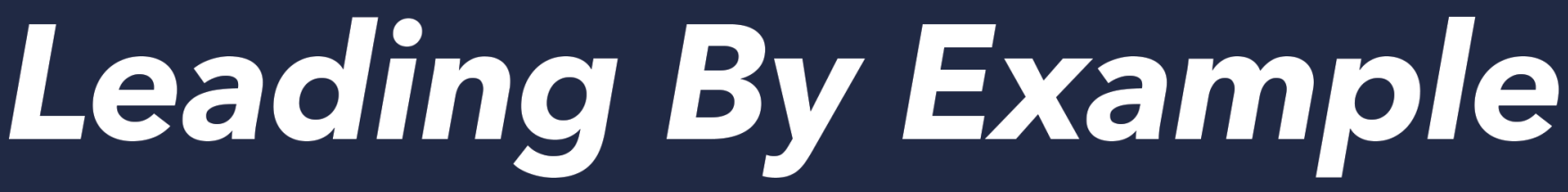

\section{\#HowWeHospitalist}

